\title{
Integration of Mirror design with Suspension System using NASA's new mirror modeling software
}

\author{
William Arnold \\ Defense Acquisitions, Inc. \\ Ryan M. Bevan \\ NASA Marshall Space Flight Center \\ Philip Stahl \\ NASA Marshall Space Flight Center \\ SUBMITTED to: \\ SPIE Conference on \\ Opto-mechanical Engineering \\ San Diego CA \\ August 25-29, 2013
}

\begin{abstract}
Advances in mirror fabrication is making very large space based telescopes possible. In the many applications, only monolithic mirrors meet the performance requirements. The existing and near-term planned heavy launch vehicles place a premium on lowest possible mass. Again, available and planned payload shroud size limits near term designs to 4 meter class mirror. Practical 8 meter and beyond designs could encourage planners to include larger shrouds if it can be proven that such mirrors can be manufactured. These two factors lower mass and larger mirrors, presents the classic optimization problem. There is a practical upper limit to how large a mirror can be supported by a purely kinematic mount system and be launched. This paper shows how the design of the suspension system and mirror blank needs to be designed simultaneously. We will also explore the concepts of auxiliary support systems, which act only during launch and disengage on orbit. We will define required characteristics of these systems and show how they can substantially reduce the mirror mass.

The AMTD project is developing and maturing the processes for future replacements for HUBBLE, creating the design tools, validating the methods and techniques necessary to manufacture, test and launch extremely large optical missions.

This paper will use the AMTD 4 meter "design point" as an illustration of the typical use of the modeler in generating the multiple models of mirror and suspension systems used during the conceptual design phase of most projects. The influence of Hexapod geometry, mirror depth, cell size and construction techniques (Exelsis Deep Core Low Temperature Fusion () versus Corning Frit Bonded ( $)$ versus Schott Pocket Milled Zerodur (C) in this particular study) are being evaluated. Due to space and time consideration we will only be able to present snippets of the study in this paper. The advances in manufacturing techniques for lightweight mirrors, such as EXELSIS deep core low temperature fusion, Corning's continued improvements in the Frit bonding process and the ability to cast large complex designs, combined with water-jet and conventional diamond
\end{abstract}

